\title{
THE RELATIONSHIP BETWEEN STRUCTURE AND IN VITRO ANTISTAPHYLOCOCCAL EFFECT OF PLANT-DERIVED STILBENES
}

\author{
TEREZA ZAKOVA ${ }^{1}$, JOHANA RONDEVALdOVA ${ }^{1}$, ANDREA BERnARdos ${ }^{2}$, \\ PREMYSL LANDA ${ }^{3}$ and LADISLAV KOKOSKA ${ }^{1 *}$ \\ ${ }^{1}$ Faculty of Tropical AgriSciences, Department of Crop Sciences and Agroforestry, Czech \\ University of Life Sciences Prague, Prague, Czech Republic \\ ${ }^{2}$ Faculty of Agrobiology, Food and Natural Resources, Department of Quality of \\ Agricultural Products, Czech University of Life Sciences Prague, Prague, Czech Republic \\ ${ }^{3}$ Laboratory of Plant Biotechnologies, Institute of Experimental Botany AS CR, Prague, \\ Czech Republic
}

(Received: 3 May 2018; accepted: 9 July 2018)

Staphylococcus aureus is a major human pathogen that is responsible for both hospital- and community-acquired infections. Stilbenes are polyphenol compounds of plant origin known to possess a variety of pharmacological properties, such as antibacterial, antiviral, and antifungal effects. This study reports the in vitro growth-inhibitory potential of eight naturally occurring stilbenes against six standard strains and two clinical isolates of $S$. aureus, using a broth microdilution method, and expressing the results as minimum inhibitory concentrations (MICs). Pterostilbene $(\mathrm{MICs}=32-128 \mu \mathrm{g} / \mathrm{ml})$, piceatannol $(\mathrm{MICs}=64-256 \mu \mathrm{g} / \mathrm{ml})$, and pinostilbene $(\mathrm{MICs}=128 \mu \mathrm{g} / \mathrm{ml})$ are among the active compounds that possess the strongest activity against all microorganisms tested, followed by 3 '-hydroxypterostilbene, isorhapontigenin, oxyresveratrol, and rhapontigenin with MICs 128-256 $\mu \mathrm{g} / \mathrm{ml}$. Resveratrol ( $\mathrm{MIC}=256 \mu \mathrm{g} / \mathrm{ml}$ ) exhibited only weak inhibitory effect. Furthermore, structure-activity relationships were studied. Hydroxyl groups at ortho-position (B-3' and $\left.-4^{\prime}\right)$ played crucial roles for the inhibitory effect of hydroxystilbene piceatannol. Compounds with methoxy groups at ring A (3'-hydroxypterostilbene, pinostilbene, and pterostilbene) produced stronger effect against $S$. aureus than their analogues (isorhapontigenin and rhapontigenin) with methoxy groups at ring $\mathrm{B}$. These findings provide arguments for further investigation of stilbenes as prospective leading structures for development of novel antistaphylococcal agents for topical treatment of skin infections.

Keywords: antimicrobial activity, natural antibacterial agents, minimum inhibitory concentration, Staphylococcus aureus, structure-activity relationships

*Corresponding author; E-mail: kokoska@ftz.czu.cz 


\section{Introduction}

Staphylococcus aureus colonizes the normal human microflora usually found on the skin and mucosa [1], and is a remarkably diverse bacterial pathogen as reflected in its capacity to cause various array of infections (e.g., pneumonia, sepsis, and skin and soft tissue infections) and food poisoning [2-4]. Antibiotics are generally applied as a conventional treatment [5]; however, $S$. aureus has acquired resistance to a majority of clinically used agents $[1,6]$. Thus, it is very likely that chemotherapy of $S$. aureus infections will become more difficult in the future [5].

Discovery of new natural antibacterial agents, which include plant-derived compounds, has regained momentum in past years as an important strategy on how to overcome the complications in the anti-infectious therapy [7]. Among these natural substances, plant stilbenes have received considerable interest over the past 20 years due to their pharmacological effects and negligible toxicity verified on various in vitro and in vivo studies, as well as a few clinical trials $[8,9]$. They occur naturally in various plant families, such as the Cyperaceae, Dipterocarpaceae, Fabaceae, Gnetaceae, Moraceae, Polygonaceae, and Vitaceae, whereas grapes and related products are considered to be the most important dietary sources of these substances [10]. In previous studies, antimicrobial effect of model stilbene resveratrol and its related structures (e.g., piceatannol, pterostilbene, trans-piceid, and trans- $\varepsilon$-viniferin) has been reported against various food and human pathogenic microorganisms, such as Acetobacter aceti, Acetobacter oeni, Bacillus cereus, Bacillus subtilis, Dekkera bruxellensis, Escherichia coli, Listeria innocua, Listeria monocytogenes, Pseudomonas fluorescens, Pseudomonas aeruginosa, Streptococcus spp., Zygosaccharomyces bailii, and Zygosaccharomyces rouxii [11-15]. The in vitro growth-inhibitory effect of (E)-3-hydroxy-5-methoxystilbene, oxyresveratrol, pterostilbene, and resveratrol has also previously been described against S. aureus and Staphylococcus epidermidis [15-18].

It is broadly recognized that the biological activity of a plant-derived compound depends on its chemical structure and that the antioxidant properties of phenolics are closely related to the types of structural terminal groups, as well as to the number and locations of hydroxyls [19]. In the case of resveratrol, it has been reported that the introduction of methoxy substitution in the place of hydroxyl groups improved the compound's antiproliferative effect by apoptosis induction and cell cycle inhibition. The more methoxy groups added, the better the antitumor activity of the compound becomes [20]. Another research showed that structural modifications of the resveratrol increase its bioavailability, while 
preserving its beneficial properties in control of atherosclerosis and heart disease [21]. Despite the reports on antistaphylococcal activity of stilbenes, the role of the functional groups at certain positions of resveratrol-related structures in S. aureus growth-inhibitory effect has not been reported, to date. For these reasons, the aim of this work is to investigate the relationship between structure and in vitro antistaphylococcal effect of various resveratrol-related compounds.

\section{Materials and Methods}

\section{Chemicals}

Isorhapontigenin (purity $>95 \%$ ), piceatannol (purity $>98 \%$ ), pinostilbene (purity $>97 \%$ ), pterostilbene (purity $>98 \%$ ), resveratrol (purity $>98 \%$ ), and rhapontigenin (purity $>98 \%$ ) were purchased from TCI EUROPE N.V. (Zwijndrecht, Netherlands); oxyresveratrol (purity $\geq 97 \%$ ) was obtained from Sigma-Aldrich (Prague, Czech Republic). 3'-hydroxypterostilbene (purity $=97 \%$ ) was received as a gift sample from the Sabinsa Corporation (NJ, USA). The dimethyl sulfoxide (Lach-ner, Neratovice, Czech Republic) has been used as solvent for stilbenes, whereas oxacillin (purity $\geq 81.5 \%$ ) and thiazolyl blue tetrazolium bromide (MTT) (purity $=98 \%$ ) (Sigma-Aldrich) were dissolved in deionized water. The potency of the compound was incorporated in the formula for the preparation of stock solutions, according to EUCAST [22].

\section{Bacterial strains and growth media}

The antimicrobial activity was evaluated against six American Typical Culture Collection strains of $S$. aureus (25923, 29213, 43300, 33591, 33592, and BAA 976) purchased from Oxoid (Basingstoke, UK). Two clinical isolates of $S$. aureus (KI1 and KI2) were obtained from the Motol University Hospital, Prague, Czech Republic. Mueller-Hinton broth (Oxoid) was used as the cultivation medium. The identification of clinical isolates was performed by matrixassisted laser desorption/ionization time-of-flight mass spectrometry as it is described in Rondevaldova et al. [23].

\section{Antimicrobial assay}

The in vitro antimicrobial activity was determined by the broth microdilution method using 96-well microtiter plates according to CLSI guidelines [24], slightly modified according to the recommendations previously proposed for 
effective assessment of the anti-infective potential of natural products [25]. Samples were twofold diluted in a range of $0.5-512 \mu \mathrm{g} / \mathrm{ml}$ and inoculated with bacterial suspension with concentration $5 \times 10^{5} \mathrm{CFU} / \mathrm{ml}$. Microtiter plates were incubated at $37{ }^{\circ} \mathrm{C}$ for $24 \mathrm{~h}$ and bacterial growth was then measured spectrophotometrically as turbidity using a Multimode Reader Cytation 3 (BioTek Instruments, Winooski, VT, USA) at $405 \mathrm{~nm}$. The MICs were expressed as the lowest concentrations, which showed at least $\geq 80 \%$ reduction of microorganisms' growth compared to that of the compound-free growth control. In order to confirm optical density measurement results, MTT was added to each well and afterward the viability of bacteria was checked visually. The results corresponding to both spectrophotometric measurement and MTT assay were used for calculation of MIC values. The assay was performed as three independent experiments each carried out in triplicate and median/modal values, which were used for final MICs determination. Oxacillin was used as the positive antibiotic reference control.

\section{Results}

In this study, all plant-derived stilbenes (chemical structures shown in Figure 1) exhibited certain degree of in vitro growth-inhibitory activity against

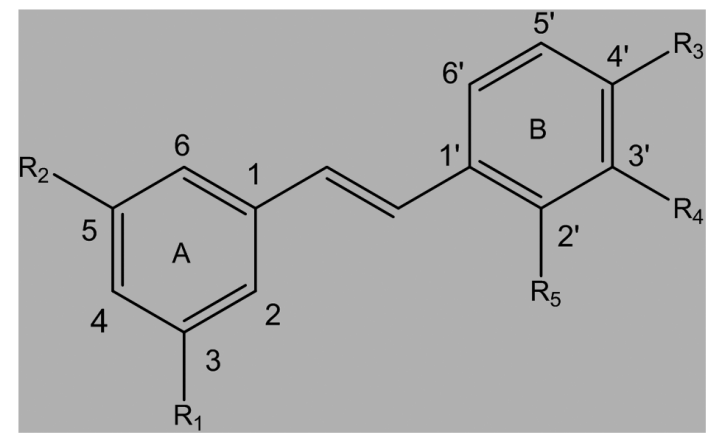

\begin{tabular}{lccccc}
\hline Name & $\mathrm{R} 1$ & $\mathrm{R} 2$ & $\mathrm{R} 3$ & $\mathrm{R} 4$ & $\mathrm{R} 5$ \\
\hline 3'-hydroxypterostilbene & $\mathrm{OCH}_{3}$ & $\mathrm{OCH}_{3}$ & $\mathrm{OH}$ & $\mathrm{OH}$ & $\mathrm{H}$ \\
Isorhapontigenin & $\mathrm{OH}$ & $\mathrm{OH}$ & $\mathrm{OH}$ & $\mathrm{OCH}_{3}$ & $\mathrm{H}$ \\
Oxyresveratrol & $\mathrm{OH}$ & $\mathrm{OH}$ & $\mathrm{OH}$ & $\mathrm{H}$ & $\mathrm{OH}$ \\
Piceatannol & $\mathrm{OH}$ & $\mathrm{OH}$ & $\mathrm{OH}$ & $\mathrm{OH}$ & $\mathrm{H}$ \\
Pinostilbene & $\mathrm{OH}$ & $\mathrm{OCH}_{3}$ & $\mathrm{OH}$ & $\mathrm{H}$ & $\mathrm{H}$ \\
Pterostilbene & $\mathrm{OCH}_{3}$ & $\mathrm{OCH}_{3}$ & $\mathrm{OH}$ & $\mathrm{H}$ & $\mathrm{H}$ \\
Resveratrol & $\mathrm{OH}$ & $\mathrm{OH}$ & $\mathrm{OH}$ & $\mathrm{H}$ & $\mathrm{H}$ \\
Rhapontigenin & $\mathrm{OH}$ & $\mathrm{OH}$ & $\mathrm{OCH}_{3}$ & $\mathrm{OH}$ & $\mathrm{H}$ \\
\hline
\end{tabular}

Figure 1. Chemical structures of stilbenes tested 
Table I. In vitro growth-inhibitory effect of stilbenes against $S$. aureus

\begin{tabular}{lcccccccc}
\hline & \multicolumn{7}{c}{ Strain tested/MIC $(\mu \mathrm{g} / \mathrm{ml})$} \\
\cline { 2 - 8 } & ATCC & ATCC & ATCC BAA & ATCC & ATCC & ATCC & \\
Compound & 43300 & 25923 & 976 & 29213 & 33591 & 33592 & KI1 & KI2 \\
\hline 3'-hydroxypterostilbene & 256 & 128 & 256 & 128 & 128 & 128 & 256 & 256 \\
Isorhapontigenin & 128 & 256 & 256 & 256 & 256 & 256 & 256 & 256 \\
Oxyresveratrol & 256 & 256 & 256 & 256 & 256 & 256 & 256 & 256 \\
Piceatannol & 64 & 64 & 64 & 64 & 64 & 64 & 64 & 256 \\
Pinostilbene & 128 & 128 & 128 & 128 & 128 & 128 & 128 & 128 \\
Pterostilbene & 32 & 32 & 32 & 32 & 64 & 32 & 32 & 64 \\
Resveratrol & $>512$ & 256 & $>512$ & $>512$ & $>512$ & 256 & $>512$ & $>512$ \\
Rhapontigenin & 256 & 256 & 256 & 256 & 256 & 128 & 256 & 256 \\
Oxacillin & 16 & 0.125 & 8 & 0.125 & 128 & 64 & 1 & 16 \\
\hline
\end{tabular}

Note: MIC: minimum inhibitory concentration; ATCC: American type culture collection; KI: clinical isolates.

*Represents reference control.

at least two out of the eight tested S. aureus strains (Table I). With the exception of one standard strain and one clinical isolate sensitive to pterostilbene at MIC $64 \mu \mathrm{g} / \mathrm{ml}$, this compound possessed the strongest antistaphylococcal effect against all strains with MIC $32 \mu \mathrm{g} / \mathrm{ml}$. This result corresponds to the findings previously published by Ishak et al. [16] who described MIC $31.25 \mu \mathrm{g} / \mathrm{ml}$ for two standard S. aureus strains. Similarly, in our tests, piceatannol inhibited growth of all strains at MIC $64 \mu \mathrm{g} / \mathrm{ml}$ except one clinical isolate $(\mathrm{MIC}=256 \mu \mathrm{g} / \mathrm{ml})$. Pinostilbene showed moderate activity with the same MIC value of $128 \mu \mathrm{g} / \mathrm{ml}$ for all tested strains, followed by $3^{\prime}$-hydroxypterostilbene, isorhapontigenin, and rhapontigenin with MIC ranging from 128 to $256 \mu \mathrm{g} / \mathrm{ml}$. Oxyresveratrol was active against all tested strains with MIC $256 \mu \mathrm{g} / \mathrm{ml}$. Our findings on oxyresveratrol and rhapontigenin are in accordance with previous studies reporting their moderate antistaphylococcal activity against standard strains. Resveratrol exhibited inhibitory effect $(\mathrm{MIC}=256 \mu \mathrm{g} / \mathrm{ml})$ only against two standard strains, which is consistent with previous reports showing that resveratrol itself has antibacterial effect at high concentrations [26]. Despite the fact that the antistaphylococcal activity of pterostilbene, resveratrol, oxyresveratrol, and rhapontigenin has previously been described, this study brings new data on growth-inhibitory effect of 3'-hydroxypterostilbene, isorhapontigenin, piceatannol, and pinostilbene against $S$. aureus. As far as the relationship between chemical structure of stilbene compounds and their antistaphylococcal effects is considered, our results suggest that the position and the number of hydroxyl and methoxy groups in rings A and B (respectively) are important for activity of stilbene compounds. Although some results differ in one- or two-dilution range, the MICs presented in this work are the 
median/modal values obtained from three independent experiments performed in triplicate and thus their values are significantly different.

\section{Discussion}

Among the group of hydroxystilbenes, piceatannol, which contained hydroxyl groups at position B-3' and $-4^{\prime}$, possessed the strongest growth-inhibitory effect against $S$. aureus. In contrast, resveratrol that produces the lowest antistaphylococcal effect has hydroxyl group on B-4' only. These results indicate that the more hydroxyl groups the stilbenes have, the stronger activity they exhibit. This is in accordance with the evidence that increased hydroxylation of phenolic compounds results in their increased toxicity to microorganisms [27]. In addition, Murias et al. [28] showed that tetrahydroxy stilbene analogues (e.g., piceatannol) have several 1,000-fold higher antiradical activities than trihydroxystilbene resveratrol. These findings suggest that increased number of hydroxyl groups on the ring structure leads to higher biological activity. However, both tetrahydroxy stilbenes, oxyresveratrol and piceatannol, significantly differ in their antistaphylococcal effects. This data suggests that not only the number of hydroxyl groups of stilbenes plays a key role in their biological effects, but also their position does. This is in correspondence with Cai et al. [29], who reported that number and location of the hydroxyl groups influenced stilbenes radical scavenging activity. As all hydroxystilbenes in this study have the same number and position of hydroxyl groups in ring A, we assume that the structure of ring B plays an important role in antistaphylococcal potential of stilbenes, which is in accordance with Tang et al. [30] who described that free radical scavenging activity of resveratrol analogues mainly depends on the hydroxyl group at ring B-4' rather than position at the ring A. As it can be observed from the results, compounds with only one hydroxyl group in ring B are less effective than the compounds with two groups. Resveratrol with hydroxyl group at position B-4' exhibited no or negligible antistaphylococcal activity. Whereas, piceatannol with hydroxyl groups at ortho-position (B-3' and $-4^{\prime}$ ) was more active than oxyresveratrol with hydroxyl groups at metaposition $\left(\mathrm{B}-2^{\prime}\right.$ and $\left.-4^{\prime}\right)$. These results indicate that ortho-dihydroxy groups in stilbene structure seem to be crucial for antistaphylococcal effect. This observation is in correspondence with previously published reports describing orthodihydroxy groups as the most important structural feature of high biological activity for phenolic compounds (e.g., stilbenes scavenging radicals) [29]. In addition, the increasing effect of antibacterial activity due to the presence of orthodihydroxy groups in structure of selected various classes of polyphenols, such as isoflavones, has also previously been proposed [31]. 
Pterostilbene, a dimethylated analogue of resveratrol with methoxy groups at positions A-3, -5 and hydroxyl group on B-4', possessed the strongest antistaphylococcal effect within all tested compounds. In general, a presence of methylated hydroxyphenyl groups in pterostilbene structure is known to increase its biological activity [32]. According to our results, compounds with methoxy groups at the ring A (3'-hydroxypterostilbene, pinostilbene, and pterostilbene) produced stronger activity against $S$. aureus than their analogues with methoxy groups at the ring B (isorhapontigenin and rhapontigenin), which suggest the important role of ring A methylation in the antistaphylococcal effect of stilbenes. Nevertheless, the presence of methoxy groups in the ring B has also previously been observed to enhance biological activity of resveratrol analogues [30]. Considering the influence of the ring A methylation on $S$. aureus growth, pterostilbene (two methoxy groups at positions A-3 and -5) possessed the strongest inhibitory effect; however, 3'-hydroxypterostilbene with two methoxy groups on A-3, -5 was less active than monomethylated structure of pinostilbene with methoxy group on A-5. Based on these results, we hypothesize that the presence of methoxy group at position A-5 may be significant for the antistaphylococcal effect of stilbenes.

Our findings suggest that stilbenes have potential as antistaphylococcal agents; however, their use in practice is determined by their toxicological and technological properties. There are several studies showing negligible toxicity of stilbenes that are abundant in many commonly consumed foods and beverages such as berries, grapes, red wine, and peanuts [33, 34]. Nevertheless, in vivo effectiveness of stilbenes is affected by their limited bioavailability due to rapid metabolism and excretion [35]. According to Wilson et al. [35], methoxylated stilbenes are metabolized more slowly, which may have a positive effect on in vivo bioactivity. Alternatively, methoxylation may protect stilbenes from metabolic modification and excretion, thereby increasing their biostability and bioavailability. In this regard, methylated structures such as pterostilbene seem to be more promising antistaphylococcal agents than the hydroxystilbenes. In addition, it has been observed that the topical administration facilitates bioavailability of pterostilbene in skin and plasma of hairless mice [36], which suggests this compound as a promising leading structure for the development of novel antistaphylococcal agents, especially for the treatment of staphylococcal skin infections. However, more detailed toxicological and microbiological studies should be determined before their practical use can be considered.

In summary, plant-derived stilbenes exhibited significant in vitro antistaphylococcal effect, specifically pterostilbene, piceatannol, and pinostilbene produced the strongest growth-inhibitory activity against all $S$. aureus strains tested. In addition, the results of the structure-activity relationship analysis suggest the 
important role of the position and the number of hydroxyl and methoxy groups in the resveratrol analogues and denote hydroxyl groups at ortho-position (B-3' and $-4^{\prime}$ ) or two methoxy groups at positions A-3 and -5 as significant supposition for the antistaphylococcal effect.

\section{Acknowledgements}

This work was financially supported by grants from the Internal Grant Agency of the Faculty of Tropical AgriSciences (IGA 20175020), by the Grant Agency of the Czech University of Life Sciences Prague (CIGA 20175001), and by the Czech Science Foundation (GA16-07193S). The authors are grateful to Nicolas Paul Wilson for his final linguistic revision of the English text.

\section{Conflict of Interest}

The authors declare no conflict of interest.

\section{References}

1. Lin, J., Lin, D., Xu, P., Zhang, T., Ou, Q., Bai, C., Yao, Z.: Non-hospital environment contamination with Staphylococcus aureus and methicillin-resistant Staphylococcus aureus: Proportion meta-analysis and features of antibiotic resistance and molecular genetics. Environ Res 150, 528-540 (2016).

2. Smeltzer, M. S.: Staphylococcus aureus pathogenesis: The importance of reduced cytotoxicity. Trends Microbiol 24, 681-682 (2016).

3. Foster, T. J.: Staphylococcus aureus. J Clin Invest 114, 1693-1696 (2004).

4. Iwatsuki, K., Yamasaki, O., Morizane, S., Oono, T.: Staphylococcal cutaneous infections: Invasion, evasion and aggression. J Dermatol Sci 42, 203-214 (2006).

5. Oyama, K., Kawada-Matsuo, M., Oogai, Y., Hayashi, T., Nakamura, N., Komatsuzawa, H.: Antibacterial effects of glycyrrhetinic acid and its derivatives on Staphylococcus aureus. PLoS One 11, 1-17 (2016).

6. Kurokawa, K., Takahashi, K., Lee, B. L.: The staphylococcal surface-glycopolymer wall teichoic acid (WTA) is crucial for complement activation and immunological defense against Staphylococcus aureus infection. Immunobiology 221, 1091-1101 (2016).

7. Silva, F., Nerín, C., Domingues, F. C.: Stilbene phytoallexins inclusion complexes: A natural-based strategy to control foodborne pathogen Campylobacter. Food Control 54, 66-73 (2015).

8. Basri, D. F., Xian, L. W., Abdul Shukor, N. I., Latip, J.: Bacteriostatic antimicrobial combination: Antagonistic interaction between epsilon-viniferin and vancomycin against methicillin-resistant Staphylococcus aureus. Biomed Res Int 2014, 461756 (2014).

9. Sirerol, J. A., Rodríguez, M. L., Mena, S., Asensi, M. A., Estrela, J. M., Ortega, A. L.: Role of natural stilbenes in the prevention of cancer. Oxid Med Cell Longev 2016, 3128951 (2016). 
10. Peng, S. C., Cheng, C. Y., Sheu, F., Su, C. H.: The antimicrobial activity of heyneanol A extracted from the root of Taiwanese wild grape. J Appl Microbiol 105, 485-491 (2008).

11. Kumar, S., Siji, J., Nambisan, B., Mohandas, C.: Activity and synergistic interactions of stilbenes and antibiotic combinations against bacteria in vitro. World J Microbiol Biotechnol 28, 3143-3150 (2012).

12. Yim, N. H., Ha, D. T., Trung, T. N., Kim, J. P., Lee, S. M., Na, M. K., Jung, H. J., Kim, H. S., Kim, Y. H., Bae, K. H.: The antimicrobial activity of compounds from the leaf and stem of Vitis amurensis against two oral pathogens. Bioorganic Med Chem Lett 20, 1165-1168 (2010).

13. Ferreira, S., Domingues, F.: The antimicrobial action of resveratrol against Listeria monocytogenes in food-based models and its antibiofilm properties. J Sci Food Agric 96, 4531-4535 (2016).

14. Pastorkova, E., Zakova, T., Landa, P., Novakova, J., Vadlejch, J., Kokoska, L.: Growth inhibitory effect of grape phenolics against wine spoilage yeasts and acetic acid bacteria. Int J Food Microbiol 161, 209-213 (2013).

15. Kabir, M. S., Engelbrecht, K., Polanowski, R., Krueger, S. M., Ignasiak, R., Rott, M., Schwan, W. R., Stemper, M. E., Reed, K. D., Sherman, D., Cooka, J. M., Monteb, A.: New classes of Gram-positive selective antibacterials: Inhibitors of MRSA and surrogates of the causative agents of anthrax and tuberculosis. Bioorganic Med Chem Lett 18, 5745-5749 (2008).

16. Ishak, S. F., Ghazali, A. R., Zin, N. M., Basri, D. F.: Pterostilbene enhanced anti-methicillin resistant Staphylococcus aureus (MRSA) activity of oxacillin. Am J Infect Dis 12, 1-10 (2016).

17. Moran, A., Gutierrez, S., Martinez-Blanco, H., Ferrero, M. A., Monteagudo-Mera, A., Rodriguez-Aparicio, L. B.: Non-toxic plant metabolites regulate Staphylococcus viability and biofilm formation: A natural therapeutic strategy useful in the treatment and prevention of skin infections. Biofouling 30, 1175-1182 (2014).

18. Joung, D. K., Mun, S. H., Choi, S. H., Kang, O. H., Kim, S. B., Lee, Y. S., Zhou, T., Kong, R., Choi, J. G., Shin, D. W., Kim, Y. C., Lee, D. S., Kwon, D. Y.: Antibacterial activity of oxyresveratrol against methicillin-resistant Staphylococcus aureus and its mechanism. Exp Ther Med 12, 1579-1584 (2016).

19. Xie, P., Huang, L., Zhang, C., Zhang, Y.: Phenolic compositions, and antioxidant performance of olive leaf and fruit (Olea europaea L.) extracts and their structure-activity relationships. J Funct Foods 16, 460-471 (2015).

20. Chen, Y., Hu, F., Gao, Y., Jia, S., Ji, N., Hua, E.: Design, synthesis, and evaluation of methoxylated resveratrol derivatives as potential antitumor agents. Res Chem Intermed 41, 2725-2738 (2015).

21. Ferrer, P., Asensi, M., Segarra, R., Ortega, A., Benlloch, M., Obrador, E., Varea, M. T., Asensio, G., Jordá, L., Estrela, J. M.: Association between pterostilbene and quercetin inhibits metastatic activity of B16 Melanoma. Neoplasia 7, 37-47 (2005).

22. European committee for antimicrobial susceptibility testing (EUCAST): Determination of minimum inhibitory concentrations (MICs) of antibacterial agents by broth dilution. Clin Microbiol Infect 9, ix-Xv (2003).

23. Rondevaldova, J., Hummelova, J., Tauchen, J., Kokoska, L.: In vitro antistaphylococcal synergistic affect of isoflavone metabolite demethyltexasin with amoxicillin and oxacillin. Microb Drug Resist 24, 24-29 (2018). 
24. Clinical and Laboratory Standards Institute (CLSI): Methods for Dilution Antimicrobial Susceptibility Tests for bacteria that grow aerobically; 8th ed. Approved Standard M07-A8. CLSI, Wayne, PA, 2009.

25. Cos, P., Vlietinck, A. J., Berghe, D. V., Maes, L.: Anti-infective potential of natural products: How to develop a stronger in vitro "proof-of-concept". J Ethnopharmacol 106, 290-302 (2006).

26. Su, Y., Ma, L., Wen, Y., Wang, H., Zhang, S.: Studies of the in vitro antibacterial activities of several polyphenols against clinical isolates of methicillin-resistant Staphylococcus aureus. Molecules 19, 12630-12639 (2014).

27. Evans, S. M., Cowan, M. M.: Plant products as antimicrobial agents. Cosmet Sci Technol Ser 31, 205-232 (2006).

28. Murias, M., Jäger, W., Handler, N., Erker, T., Horvath, Z., Szekeres, T., Nohl, H., Gille, L.: Antioxidant, prooxidant and cytotoxic activity of hydroxylated resveratrol analogues: Structure-activity relationship. Biochem Pharmacol 69, 903-912 (2005).

29. Cai, Y.-Z., Sun, M., Xing, J., Luo, Q., Corke, H.: Structure-radical scavenging activity relationships of phenolic compounds from traditional Chinese medicinal plants. Life Sci $\mathbf{7 8}$, 2872-2888 (2006).

30. Tang, F., Xie, Y., Cao, H., Yang, H., Chen, X., Xiao, J.: Fetal bovine serum influences the stability and bioactivity of resveratrol analogues: A polyphenol-protein interaction approach. Food Chem 219, 321-328 (2017).

31. Hummelova, J., Rondevaldova, J., Balastikova, A., Lapcik, O., Kokoska, L.: The relationship between structure and in vitro antibacterial activity of selected isoflavones and their metabolites with special focus on antistaphylococcal effect of demethyltexasin. Lett Appl Microbiol 60, 242-247 (2015).

32. Chong, J., Poutaraud, A., Hugueney, P.: Metabolism and roles of stilbenes in plants. Plant Sci 177, 143-155 (2009).

33. Shi, Y. W., Wang, C. P., Liu, L., Liu, Y. L., Wang, X., Hong, Y., Li, Z., Kong, L. D.: Antihyperuricemic and nephroprotective effects of resveratrol and its analogues in hyperuricemic mice. Mol Nutr Food Res 56, 1433-1444 (2012).

34. McCormack, D., McFadden, D.: Pterostilbene and cancer: Current review. J Surg Res 173, e53-e61 (2012).

35. Wilson, M., Rimando, A., Wolkow, C.: Methoxylation enhances stilbene bioactivity in Caenorhabditis elegans. BMC Pharmacol 8, 15 (2008).

36. Sirerol, J. A., Feddi, F., Mena, S., Rodriguez, M. L., Sirera, P., Aupí, M., Pérez, S., Asensi, M., Ortega, A., Estrela, J. M.: Free radical biology and medicine topical treatment with pterostilbene, a natural phytoalexin, effectively protects hairless mice against UVB radiation-induced skin damage and carcinogenesis. Free Radic Biol Med 85, 1-11 (2015). 